\title{
User-friendly MES Interfaces: Recommendations for an AI-based Chatbot Assistance in Industry 4.0 Shop Floors
}

\author{
Soujanya Mantravadi 10000-0001-9382-8314], Andreas Dyrøy Jansson 2[0000-0001-5116-6041], \\ Charles Møller ${ }^{10000-0003-0251-3419] ~}$ \\ ${ }^{1}$ Department of Materials \& Production, Aalborg University, Aalborg, Denmark \\ smemp.aau.dk, charles@mp.aau.dk \\ ${ }^{2}$ Department of Computer Science and Computational Engineering, UiT - Arctic University of \\ Norway, Narvik, Norway \\ andreas.d.jansson@uit.no
}

\begin{abstract}
The purpose of this paper is to study an Industry 4.0 scenario of 'technical assistance' and use manufacturing execution systems (MES) to address the need for easy information extraction on the shop floor. We identify specific requirements for a user-friendly MES interface to develop (and test) an approach for technical assistance and introduce a chatbot with a prediction system as an interface layer for MES. The chatbot is aimed at production coordination by assisting the shop floor workforce and learn from their inputs, thus acting as an intelligent assistant. We programmed a prototype chatbot as a proof of concept, where the new interface layer provided live updates related to production in natural language and added predictive power to MES. The results indicate that the chatbot interface for MES is beneficial to the shop floor workforce and provides easy information extraction, compared to the traditional search techniques. The paper contributes to the manufacturing information systems field and demonstrates a human-AI collaboration system in a factory. In particular, this paper recommends the manner in which MES based technical assistance systems can be developed for the purpose of easy information retrieval.
\end{abstract}

Keywords: AI Applications, Manufacturing, Chatbot

\section{Introduction}

Although manufacturing execution systems have been critical information systems for production planning and control among manufacturing practitioners, their interaction with humans in Industry 4.0 needs further understanding in theory and in practice. MES as an information system has come a long way. It was first introduced in the 70 s to assist the online management of production execution. Later in the 90s, it emerged as a powerful software tool to replace paper-based activities of manufacturing operations management (MOM) [1]. Over the years, it became a critical tool for a manufacturing enterprise as it acquired additional functionalities for automation of information exchange due to computer advancements and shop floor systems integration projects [2]. 
For future factories, studying the enhancements for MES in the light of Industry 4.0 context can bring new opportunities that were not identified before. The Industry 4.0 paradigm is predominantly information-centric and guides manufacturing enterprises to assess their future needs and acquire digital capabilities. Industry 4.0 is an IT-driven enabler of smart factories [3] with the design principles of [4]: (a) Interconnection (b) Information transparency (c) Decentralized decision-making (d) Technical assistance. In this paper, we focus on the design principle of 'technical assistance' for the factory workforce, which is about humans receiving support from assistant systems for production-related tasks [5][4].

Artificial intelligence capability is an asset for manufacturing because the digital age promises hardware with high processing power combined with the vast amounts of available real-time production data generated by MES. Analogous to natural intelligence, an AI system is able to learn from the experience, where data equals experience. A larger set of training production data makes for a robust decision-making system for operations management. AI applications in the manufacturing field are not new and they are widely studied to manage uncertainty, complexity and dynamic changes in the manufacturing systems [6][7]. On this premise, combining MES with AI can drastically improve automated decision-making capabilities and the workforce can benefit from this situation.

Furthermore, intuitive assistant systems designed based on AI techniques can help the workforce make informed decisions in enterprises [8][9]. Motivated by the need to study their potential industrial engineering applications, we emphasize on user aspects and design a chatbot for the information system (MES). We apply techniques such as natural language processing and artificial neural networks, which are key parts in making a chatbot 'intelligent' to collaborate with the MES user (intelligence means predictive power in this paper). Hence, our research objective is to use MES to accommodate AI-based technical assistance.

Section 2 presents the related theoretical work and Section 3 describes the research methodology. Findings are presented in Section 4 and discussed in Section 5. Finally, conclusions are drawn in Section 6.

\section{Related work}

\subsection{MES Interfaces}

Even though MES is meant to interact with humans on the shop floor for smoother production management, this aspect has not been studied extensively in the academic literature. The design purpose of MES is to support human decision making for activities related to manufacturing operations management. MES does this by making the production data accessible in real-time. Due to this, it is deemed a 'manufacturing cockpit' [10]. There have been studies to improve processes by analyzing real-time production data from MES [2], but not many of them focus on enhancing the efficiency of shop floor operations by deploying conversational assistants (with predictive power) as collaborating agents. 
Production data acquisition was formerly needed to calculate machine utilization, whereas, current day's factories need it for real-time process adjustments. Production data is valuable for manufacturing enterprises to meet future market demands for product variability and faster deliveries. Timely availability of production data will boost the existing operational procedures related to work scheduling, customer interaction, order fulfillment, toolmaking, costing and supplier interaction, etc.

MES can have different user groups with customized interfaces and modules for each group. As an example, the management personnel can have access to the monitoring module and might be able to calculate key performance indicators (KPIs) from the acquired data, whereas the machine operators might have access to its planning module. The table below has a broad classification of MES users and their possible role if MES is combined with AI-based prediction systems for information exchange.

Table 1. MES User on the shop floor.

\begin{tabular}{lll}
\hline Category & Existing role & Possible engagement \\
\hline $\begin{array}{l}\text { Management } \\
\text { Personnel }\end{array}$ & Monitoring the production process & $\begin{array}{l}\text { foresee inventory shortage and manage } \\
\text { orders * }\end{array}$ \\
\hline $\begin{array}{l}\text { Operator } \\
\text { personnel }\end{array}$ & $\begin{array}{l}\text { Production planning, scheduling, } \\
\text { dispatching, tracking }\end{array}$ & $\begin{array}{l}\text { Anomaly detection and take corrective } \\
\text { action on the production line [11] }\end{array}$ \\
\hline
\end{tabular}

* Focus of this study

User-friendly MES interfaces provide easy access to data, thus supporting human decisions in manufacturing operations. There are standards such as ISA 101 to suggest the best practices of human-machine interfacing in manufacturing [12]. ISA 101 covers menu hierarchies, screen navigation conventions, security methods and electronic signature attributes, pop-up conventions, configuration interfaces to databases, servers, etc.

In this regard, modern MES also comes with dashboards that display production, process insights using data visualization tools. They enhance the human-machine interface and particularly benefit management personnel that performs process data mining and monitoring. Some MES vendors also offer the dashboards as apps. However, these data access tools are oftentimes expensive and non-conversational.

\subsection{Idiosyncrasies of Shop Floor}

Manufacturing control and management had a variety of applications on the shop floor. Industry practices around it were mostly about shop floor operators manually modifying the processes using ad hoc methods. There was a minimal need for re-planning or on-line measurement and real-time feedback control [13]. However, with changing manufacturing requirements (such as demand for product variability, shorter time to market, engineer-to-order production strategies and real-time factory scheduling) the future demands on in-process management measurement and feedback control cannot be met with traditional practices. 
Over the years, MES successfully served as a factory database [14] but faced resistance due to its inflexibility, monolithic architecture and for being an expensive investment. With the ever-increasing volumes of logged production data, manufacturing enterprises might reconsider MES for building a foundation for their future data management initiatives. Considering the importance of data visibility, we argue that intelligent planning and control concepts must be infused in MES to predict and coordinate production activities. Due to this, the MES user can engage in dynamic manufacturing operations with rapid responsiveness.

\subsection{Potentials in Chatbots}

Context-sensitive user interfaces and real-time learning assistants are some forms of interactions in the cyber-physical world. These technologies simplify the complexity of workers with information and interaction possibilities [5]. Chatbots can be used as conversational information systems [15] and are promising for filtering and processing information. However, chatbots are a nascent technology and their application in enterprises is still not well understood [15]. Chatbots use artificial intelligence and combining them with MES for human-machine interaction can complement the production workforce by demanding flexibility and creativity [16].

A chatbot for MES provides a more natural interaction platform compared to a traditional MES human interface. Being an automated system for enhancing human-computer interaction, chatbots can parse MES user input in the form of natural language and generate an appropriate response text. This may be done in several ways. The simplest is by providing the bot with a database of questions and answers. This approach works well for knowledge bases and static information but falls short in a dynamic environment [17]. Chatbot as an interface layer can also serve as a replacement for MES dashboards or can complement it. A chatbot for MES caters to the digitalization of MES, which is needed for building Industry 4.0 capabilities in a factory [18]. Owing to these principles, we hypothesize:

H) A chatbot interface is a user-centric design enhancement for MES and it serves to monitor manufacturing operations by easy retrieval of information on demand.

\section{Approach}

We used a combination of empirical study and an experiment for this paper. First, we conducted a selective literature review using the databases Google Scholar and Scopus, with an emphasis on the publications from 2015-2019. The keywords 'MES', 'manufacturing operations management', 'technical assistance in Industry 4.0', 'chatbots' etc were searched and Mendeley was used to manage the references. The review results provided knowledge on MES functionalities and its usefulness for coordinating manufacturing operations when combined with conversational virtual assistants. Based on the results, we identified applicable AI techniques. The literature review also helped in driving the empirical study. 
Second, to ensure the relevance aspect of the MES design problem and to determine the requirements of the manufacturing industry, empirical evidence on the existing state of operations management technologies were needed. Therefore, we studied four companies to understand business and technological drivers to design technical assistance using MES. Due to commercial confidentiality, they are called Alpha, Beta, Gamma, and Delta. We conducted empirical research, where data triangulation was done using semi-structured interviews, field studies, archival documents, and industry reports.

In the following table, Finding 1 concern the company's drive for choosing MES and Finding 2 concerns the assistance requirements for MES users:

Table 2. Summary of the collected qualitative data.

\begin{tabular}{|c|c|c|}
\hline Company & Data type & Description \\
\hline \multirow{4}{*}{ Alpha } & Size & $>10,000$ employees \\
\hline & Industry & Dairy \\
\hline & Finding 1 & $\begin{array}{l}\text { MES to develop the Industrial IoT ecosystem in the company using enter- } \\
\text { prise systems }\end{array}$ \\
\hline & Finding 2 & $\begin{array}{l}\text { The requirement to provide flexibility and visibility to the shop-floor per- } \\
\text { sonnel using dashboards based on real-time production data from MES }\end{array}$ \\
\hline \multirow{5}{*}{ Beta } & Size & $>10,000$ employees \\
\hline & Industry & Meat processing \\
\hline & Finding 1 & MES to streamline processes and gain competitiveness \\
\hline & Finding 2 & $\begin{array}{l}\text { The requirement to react to supply/demand problems using timely availa- } \\
\text { ble production data }\end{array}$ \\
\hline & Size & $>10,000$ employees \\
\hline \multirow{3}{*}{ Gamma } & Industry & Electrical equipment \\
\hline & Finding 1 & MES to secure manufacturing intelligence \\
\hline & Finding 2 & $\begin{array}{l}\text { The requirement for real-time monitoring and control of cross-business- } \\
\text { unit coordination }\end{array}$ \\
\hline \multirow{4}{*}{ Delta } & Size & $>10,000$ employees \\
\hline & Industry & Energy equipment \\
\hline & Finding 1 & Primarily use MES for order execution, but also learn from the acquired \\
\hline & Finding 2 & $\begin{array}{l}\text { The requirement to use technologies that can give insight into data and } \\
\text { enhance the human-machine interface (HMI). Currently, the production } \\
\text { line controller is the HMI for shop floor personnel }\end{array}$ \\
\hline
\end{tabular}

Data provided insights into the phenomenon of technical assistance for manufacturing planning and control, its dynamics and the importance of information flows in a factory. Based on the gathered requirements a representative use case of information flow for order management is identified. It falls under the category of management personnel (see Table. 1). Consequently, the design requirements were documented (see 4.1).

Lastly, as an experiment, we programmed a prototype chatbot and connected it to a simple web service endpoint, simulating a web-based MES database (we used Odoo, a cloud-based enterprise software offered as a service) of AAU Smart Production lab [19]. To create a simple demonstrator, we used AIML. AIML is a free and open-source artificial intelligence markup language and is the framework of choice for major chatbot platforms, including Pandorabots. The language is XML-based and can be used to create both simple and complex, state-aware chatbots. Using basic tags combined with pattern recognition, the chatbot was able to provide a rational response from its knowledge base [20]. Thus, we had a proof of concept for MES based technical assistance (see 4.2). The collected qualitative data also helped in evaluating the benefits of such chatbots in the manufacturing context. The following section presents the findings on the requirements of a User-friendly MES interface. 


\section{$4 \quad$ Findings}

\subsection{High-level Goals}

The literature and empirical evidence suggest that enterprises are re-considering MES to launch their future Industry 4.0 data management initiatives. Furthermore, we also noted that the role of humans shifts from machine operator to strategic decision-maker and a flexible problem-solver due to technical assistance in Industry 4.0 [4]. Therefore, we studied if the assistance systems are compatible with a typical MES system, which has the factory data access in real-time. MES focusses on 'production data collection', which is defined by IEC 62264-3 standard as an activity of gathering and managing information on work processes and production orders [2]. For this purpose, the multiple requirements of MES users are identified and their developments are presented in the figure below:

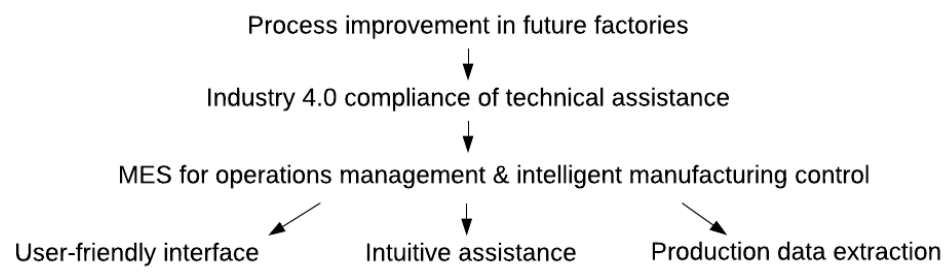

Fig. 1. Requirements breakdown for an MES based technical assistance.

Bussmann \& McFarlane summarize the future manufacturing demands as increasing complexity and continual change, where anticipated control system properties will not suffice. Futuristic operations need intelligent control and data is key to increase the responsiveness of computer control systems and to aid human operations. To achieve such intelligence, the first step for the system is to have context-awareness, meaning fewer assumptions are to be made on process and component behavior [21]. MES supports control actions being taken in real-time. Live access to production status can improve operational procedures of the workforce and for this purpose; the MES system can be developed with added intelligence in a manufacturing enterprise. We identified that MES can be extended with a chatbot prediction system to enhance user experience and to support human decision making.

In the changing manufacturing times, the strategy to improve customer responsiveness and reduce time to market is extremely crucial. Hence, we studied order management practices to design a system that can proactively suggest relevant information based on the previous input. We focused on the system's capability to update the state of order and anticipate delays. 


\subsection{Low-level Goals}

In a factory environment, chatbot for MES can be made 'intelligent' using machine learning techniques [22] for it to learn from the repeated queries of users. The relevant approaches are NLP and ANN (discussed further in 5.2). The proposed MES design enhancement to accommodate AI-based technical assistance consists of:

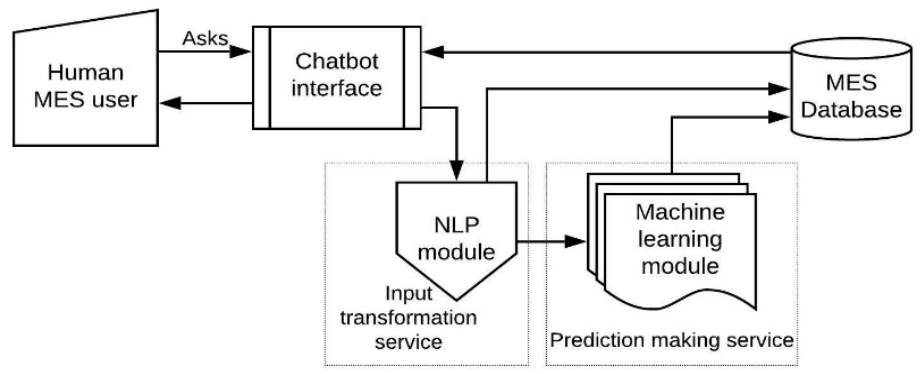

Fig. 2. MES based Technical assistance system.

In Fig. 2 above, the relationship between various parts of the system is shown. Here, a human user interacts with a chatbot interface and may ask about the current state of affairs using natural language. This interaction may be represented using the following algorithm (see Fig. 3).

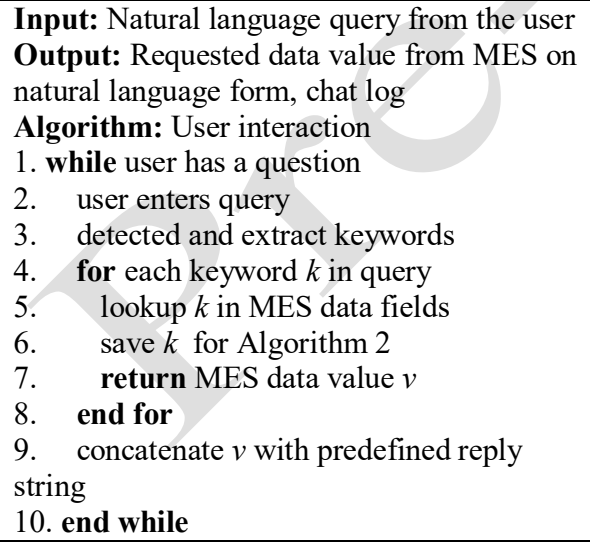

Input: Chat $\log D$ where $D_{\text {size }}>1$

Output: Training set for prediction system Algorithm: Creating training set for prediction system

1. for each query $q$ in $D$

2. for each keyword $k$ in $q$

3. $\operatorname{map} k$ to numerical value $(0,1]$

4. add $k$ to training set - input vector

5. end for

6. for each keyword $k^{\prime}$ in $q+1$

7. $\operatorname{map} k$ ' to numerical value $(0,1]$

8. add $k$ ' to training set - output vector

9. end for

10 . end for

$((q+1)$ is the successive query)

Fig. 3. Chatbot interaction and training algorithms.

A graphical representation of Algorithm 2 is presented in Fig. 4 below. The first known word in the list is assigned 0.1 , the last 1.0. The input vector size is set to 4 . If there are fewer keywords than this, the value 0.0 is used. If the number of keywords is greater than 4 , the most significant words according to the inverse document frequency are used. 


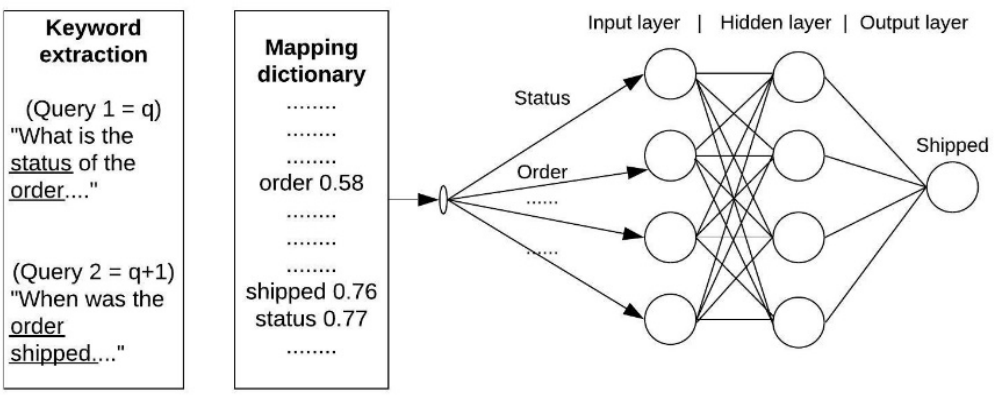

Fig. 4. Input transformation and network training.

\subsection{Output \& Assessment}

To study the fundamental differences between conventional MES user interface and MES with a chatbot user interface, the prototype is assessed based on the chatbots' ability to retrieve order information. However, the chatbot is able to retrieve any data from MES given that they are exposed through a web service. Fig. 5 shows examples of returned output. The input from the chatbot client is returned along with a message from the server.

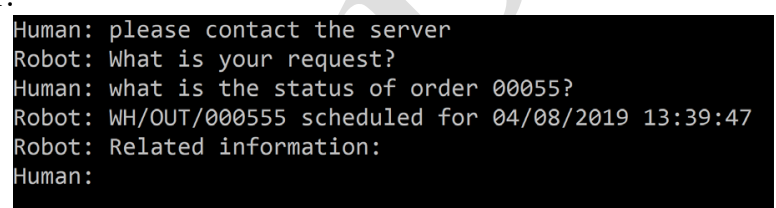

Fig. 5. Assessment of conversational MES interface.

We assess the success of MES based technical assistance system based on the ease with which user received a response without having to enter complete order ID. Our qualitative assessment suggests that this approach is easier than the regular database lookup.

The output (see Fig.5), as well as the assessment, supports the hypothesis that it is easy to retrieve production information using a chatbot interface. The collected empirical data (see Table. 2) also suggests that such retrieval process is beneficial to manufacturing. Challenges regarding the training of the system are presented in the following discussion section.

\section{Discussion}

Previous studies on enterprise systems attempted to close the gap of their integration with shop floor devices in a service-oriented way [23], but they did not focus on the MES user empowerment in the age of industrial internet. In response to the growing interest in practitioners to digitize manufacturing operations and to pursue Industry 4.0 
[4], we proposed a concept that provides assistance to the production personnel (team) to make planning and control decisions by reacting quickly. The rationale for choosing MES instead of any other information system in the factory is also explained. The next iteration of this paper is expected to investigate its impact on the existing operational procedures for process improvement. Such a study can expose the technical and organizational challenges in implementing MES based technical assistance systems.

\subsection{Shop Floors with Virtual Intelligent-assistant}

The concept of 'Internet of people' connects the workforce to the internet using interfaces. It promotes people-centric design enhancements with the principles of being social, personal and proactive [24]. In a factory's dynamic environment, a chatbot can be connected to the sensors of the physical assets on the shop floor or to external data providers to generate answers on the fly. A chatbot with a prediction system can also be connected to a database supported knowledge base [25]. Since MES is a factory database with real-time production data access and a unified functional platform of IT, we designed a 'human-AI collaboration system' around it.

A chatbot could, in theory, be connected to any data providing service. For the shop floor workforce, a chatbot creates an alternative to looking through menus and tables of MES to find the correct information and aids smoother information access [26]. Since MES is a database application with multiple user groups in a factory, designing a collaborative system (a combination of processes and software to support a group activity) around it is possible. Vision for the MES collaborative system needs further inquiry on the processes that involve group activity on the shop floor. Such an effort also aligns with the concept of 'digital workplace' that promotes virtual assistance [27].

The system may be extended to generate complex answers from the bot and to create a more helpful interface for MES. This prediction system may also be connected to other external data sources to provide additional responses relevant to shop floor activities such as materials inventory, shipping, traffic, exchange rates, etc.

\subsection{Real-time AI Techniques for MES}

Contrary to the popular belief that the application of artificial intelligence to automate workplace tasks might lead to the unemployment crisis [28], we present a situation where the advances in AI techniques are utilized to assist the workforce (see Fig. 1). We used NLP that is crucial for creating a chatbot as it enables a computer system to interpret and find the intent of the MES user's input. In this context, NLP can be implemented using the inverse document frequency algorithm to detect keywords, along with Levenshtein-distance [29] to compensate for spelling errors. This approach approximates MES user input with known chatbot vocabulary as per the dictionary mapping discussed earlier.

Enabling interaction with MES using natural language instead of navigating through complex or even unintuitive user interfaces is imagined to increase user-friendliness, especially for novice users. The user only gets the information they are requesting and do not have to be distracted by the information irrelevant to their current task. This is 
evident from Fig.4, where the user was not required to enter the complete order ID in order to get a response. The reply from the bot contains full information as retrieved from MES. This functionality is akin to a sophisticated search function, although it comes with the added benefit of flexible queries in the form of natural language. However, this also enables traditional keyword-based searches in the same interface (The NLP system we presented is available only in the English language).

Training the system. Artificial neural networks are often used for prediction systems [30][31] and the ANN in our design is trained on the transformed MES user input to suggest relevant information, which turns the bot into an intelligent assistant for MES users. Being able to get related information automatically is expected to be a helpful feature. However, the suggestions given must be evaluated for their usefulness. If the system is not properly trained, offering irrelevant or even wrong information will serve as a source of frustration. Giving feedback will help the system learn and provide appropriate suggestions. However, this should not affect the normal routines of workers. Using the time to train the system would defeat its purpose. Ideally, some person could be assigned to train and improve the accuracy of the chatbot. Training an ANN is an iterative process and is performed until the produced output is within a set error limit of the expected output. Over time, accuracy is expected to improve and less time will be needed to monitor the chatbot.

\subsection{In-house Chatbot Solution}

Deploying an MES based chatbot and its services are expected to be a major advantage (also cost-effective) compared to existing virtual assistants. Examples of this technology include Google's Assistant and Apple's Siri. Nonetheless, as they are proprietary systems by external vendors, the information must pass through their systems. For sensitive production data, this can be a major drawback. They also come packed with features that a shop floor might not require, where the focus is to get the required information as swiftly as possible. A domain-specific expert chatbot solution like the one presented in this paper will require less initial setup, as it is only connected to the internal services. Furthermore, the chatbot and its accompanying web service may further tailored to fit a specific purpose, as new and unexpected needs might arise in production. In order to realize the full potentials of the chatbot interface layer, we recommend using web-based MES because it makes it easy to scale-up, add clients and functionalities. Its architecture can be presented as:

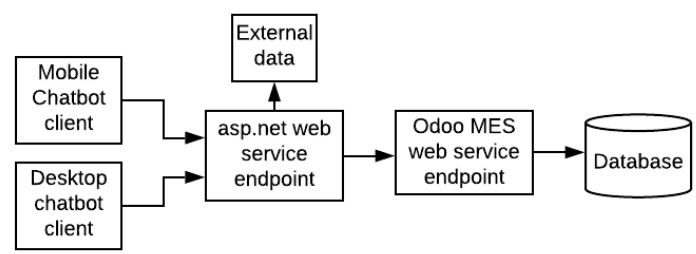

Fig. 6. Web service architecture for MES. 
Such design eliminates the traditional monolithic MES architecture and makes it flexible to develop next-generation infrastructural capabilities for the shop floor. Based on a service-oriented architecture method, a digitally enriched MES will be able to facilitate the easy integration of applications as well as enable itself to connect to the network services like the one presented in this paper.

Finally, the data gathered by the chatbot in the form of user queries may also be analyzed to get an insight into the state of production and worker satisfaction. The presented user-friendly MES interface layer demonstrated such behavior, even though the efficacy and accuracy of chatbots for data extraction remains questionable. The current study gave an example of 'order management' but the future work is expected to analyze its impact on a wider range of processes related to manufacturing operations management, based on the aforementioned design.

\section{Conclusions}

Manufacturing enterprises are reconsidering MES for implementing their future data management initiatives (see 4.1). Therefore, we attempted to digitally enrich the MES platform for technical assistance on data retrieval. The findings suggest that the intelligent chatbot for MES can assist the shop floor personnel and can aid the operator's decision making (see 4.3). Because of this design criterion, MES users could engage in dynamic operations with enhanced responsiveness. The study demonstrated a humanAI collaboration in a factory, where MES fostered unstructured collaboration as it feeds the users' responses (data) into the system for further learning. The collected empirical data also helps us in concluding that the chatbot interface for MES is highly beneficial in the manufacturing context. As a result, recommendations for AI-based chatbot assistance in Industry 4.0 shop floors were presented (see 5).

As far as we know, this is the first time an intelligent chatbot is used for MES to enable a factory as a digital workplace and to scientifically approach the Industry 4.0 design principle of 'Technical assistance'. Future work intends to add more complexities to the proposed design to test its benefits and advance the theory of manufacturing digitalization.

Acknowledgment. This research work is partially funded by the Manufacturing Academy of Denmark. The authors would like to thank the informants from the companies for sharing their knowledge.

\section{References}

1. MESA White Paper \#01: The Benefits of MES: A Report from the Field. Presented at the (1997).

2. Scholten, B.: The Road to Integration. ISA (2007).

3. Sauer, O.: Information technology for the factory of the future - State of the art and need for action. Procedia CIRP. 25, 293-296 (2014). https://doi.org/10.1016/j.procir.2014.10.041.

4. Hermann, M., Pentek, T., Otto, B.: Design principles for industrie 4.0 scenarios. Proc. Annu. Hawaii Int. Conf. Syst. Sci. 2016-March, 3928-3937 (2016).

5. Gorecky, D., Schmitt, M., Loskyll, M., Zühlke, D.: Human-machine-interaction in the industry 4.0 
era. Proc. - 2014 12th IEEE Int. Conf. Ind. Informatics, INDIN 2014. 289-294 (2014).

6. Monostori, L., Váncza, J., Kumara, S.R.T.: Agent-based systems for manufacturing. CIRP Ann. Manuf. Technol. 55, 697-720 (2006). https://doi.org/10.1016/j.cirp.2006.10.004.

7. Hatvany, J., Nemes, L.: Intelligent Manufacturing Systems- A Tentative Forecast. IFAC Proc. Vol. 11, 895-899 (1978). https://doi.org/10.1016/S1474-6670(17)66031-2.

8. Maedche, A., Morana, S., Schacht, S., Werth, D., Krumeich, J.: Advanced User Assistance Systems. Bus. Inf. Syst. Eng. 58, 367-370 (2016). https://doi.org/10.1007/s12599-016-0444-2.

9. Stoeckli, E., Uebernickel, F., Brenner, W.: Exploring Affordances of Slack Integrations and Their Actualization Within Enterprises - Towards an Understanding of How Chatbots Create Value. Proc. 51 st Hawaii Int. Conf. Syst. Sci. 2016-2025 (2018). https://doi.org/10.24251/hicss.2018.255.

10. Kletti, J.: Manufacturing Execution Systems - MES. Springer Berlin Heidelberg New York (2007).

11. Mantravadi, S., Li, C., Møller, C.: Multi-agent Manufacturing Execution System (MES): Concept, Architecture \& ML Algorithm for a Smart Factory Case. In: Scitepress. pp. 477-482 (2019). https://doi.org/10.5220/0007768904770482.

12. ISA101, Human-Machine Interfaces, https://www.isa.org/.

13. Albus, J.S.: An Intelligent Systems Architecture for Manufacturing. Int. Conf. Intell. Syst. A Semiot. Perspect. 20-23 (1996).

14. Younus, M., Hu, L., Yuqing, F., Yong, C.P.: Manufacturing execution system for a subsidiary of aerospace manufacturing industry. Proc. - 2009 Int. Conf. Comput. Autom. Eng. ICCAE 2009. 208-212 (2009). https://doi.org/10.1109/ICCAE.2009.12.

15. Wolff, R.M. Von, Hobert, S., Schumann, M.: How May I Help You ? - State of the Art and Open Research Questions for Chatbots at the Digital Workplace. Proc. Hawaii Int. Conf. Syst. Sci. 6, 95104 (2019).

16. David H. Autor, F.L., Murnane, R.J.: The Skill Content of Recent Technological Change: An Empirical Exploration. Q. J. Econ. 1279-1333 (2003).

17. Augello, A., Pilato, G., Machi, A., Gaglio, S.: An approach to enhance chatbot semantic power and maintainability: Experiences within the FRASI project. Proc. - IEEE 6th Int. Conf. Semant. Comput. ICSC 2012. 186-193 (2012). https://doi.org/10.1109/ICSC.2012.26.

18. Demartini, M., Tonelli, F., Damiani, L., Revetria, R., Cassettari, L.: Digitalization of manufacturing execution systems: The core technology for realizing future smart factories. Proc. Summer Sch. Fr. Turco. 2017-Septe, 326-333 (2017).

19. Madsen, O., Møller, C.: The AAU Smart Production Laboratory for Teaching and Research in Emerging Digital Manufacturing Technologies. Procedia Manuf. 9, 106-112 (2017).

20. Marietto, M. das G.B., de Aguiar, R.V., Barbosa, G. de O., Botelho, W.T., Pimentel, E., França, R. dos S., da Silva, V.L.: Artificial Intelligence MArkup Language: A Brief Tutorial. (2013).

21. Bussmann, S., McFarlane, D.: Rationales for Holonic Manufacturing Control. Proc. 2nd Int. Work. Intell. Manuf. Syst. 1-8 (1999).

22. Wu, Y., Wang, G., Li, W., Li, Z.: Automatic chatbot knowledge acquisition from online forum via rough set and ensemble learning. Proc. - 2008 IFIP Int. Conf. Netw. Parallel Comput. NPC 2008. 242-246 (2008). https://doi.org/10.1109/NPC.2008.24.

23. Karnouskos, S., Baecker, O., De Souza, L.M.S., Spieß, P.: Integration of SOA-ready networked embedded devices in enterprise systems via a cross-layered web service infrastructure. IEEE Int. Conf. Emerg. Technol. Fact. Autom. ETFA. 293-300 (2007).

24. Miranda, J., Mäkitalo, N., Garcia-Alonso, J., Berrocal, J., Mikkonen, T., Canal, C., Murillo, J.M.: From the Internet of Things to the Internet of People. IEEE Internet Comput. 19, 40-47 (2015).

25. Reshmi, S., Balakrishnan, K.: Implementation of an inquisitive chatbot for database supported knowledge bases. Sadhana - Indian Acad. Sci. 41, 1173-1178 (2016).

26. Carayannopoulos, S.: Using chatbots to aid transition. Int. J. Inf. Learn. Technol. 35, 118-129 (2018).

27. Lestarini, D., Raflesia, S.P., Surendro, K.: A conceptual framework of engaged digital workplace diffusion. Proceeding 2015 9th Int. Conf. Telecommun. Syst. Serv. Appl. TSSA 2015. (2016).

28. Levy, F.: Computers and populism: Artificial intelligence, jobs, and politics in the near term. Oxford Rev. Econ. Policy. 34, 393-417 (2018). https://doi.org/10.1093/oxrep/gry004.

29. Michael Gilleland: Levenshtein Distance, in Three Flavors, https://people.cs.pitt.edu/ kirk/cs1501/Pruhs/Spring2006/assignments/editdistance/Levenshtein Distance.htm.

30. Kimoto, T., Asakawa, K., Yoda, M., Takeoka, M.: Stock market prediction system with modular neural networks. 1-6 vol.1 (2002). https://doi.org/10.1109/ijenn.1990.137535.

31. Sheth, A., et al..: A Sensor Network Based Landslide Prediction System. SenSys. 280-281 (2005). 